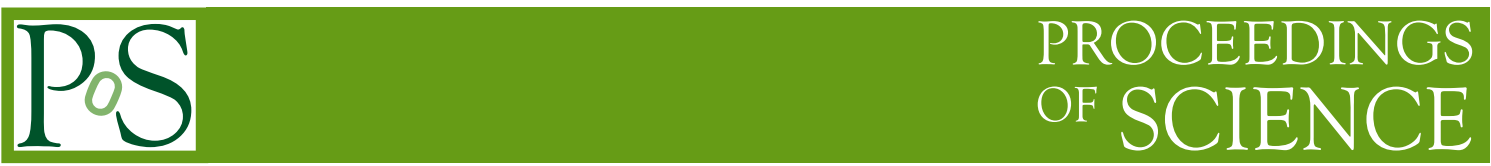

\title{
The Forward Diffractive Detector for ALICE
}

\author{
Solangel Rojas-Torres ${ }^{a, *}$ for the ALICE Collaboration \\ ${ }^{a}$ Czech Technical University in Prague, \\ Brehova 7, Prague, Czech Republic \\ E-mail: solangel.rojas.torres@cern.ch, rojassol@fjfi.cvut.cz
}

ALICE (A Large Ion Collider Experiment) is one of the four main detectors at the CERN LHC. In order to exploit the increased luminosity and interaction rate during the upcoming LHC Run 3 and 4, ALICE is now implementing a significant upgrade of its detectors and systems.

The minimum latency interaction trigger, luminosity monitoring, precision collision time, and determination of centrality and event plane for heavy-ion collisions will be provided by the Fast Interaction Trigger (FIT). FIT consists of three subsystems, among them the Forward Diffractive Detector (FDD).

In this work, we introduce the FDD, which is the upgrade of the former ALICE Diffractive (AD) detector. The FDD detector consists of two stations covering the pseudorapidity ranges of $4.7<\eta<6.3$ and $-6.9<\eta<-4.9$, respectively. This coverage allows FDD to efficiently tag diffractive and ultra-peripheral events. The stations are made of two layers of plastic scintillators, divided into four quadrants. Each quadrant has two wavelength shifting (WLS) bars connected to individual PMTs via a bundle of clear optical fibres. Among the main improvements of the FDD, with respect to the AD, are faster plastic scintillators and WLS, and the use of the newly developed front-end electronics suitable for operation both in a triggered and in a continuous readout mode. The FDD will ensure that ALICE has the large pseudorapidity coverage needed to (1) select diffractive events down to diffractive masses of a few $\mathrm{GeV} / c^{2}$, and (2) to veto particle production in the forward regions in order to obtain clean samples of ultra-peripheral events.

The Eighth Annual Conference on Large Hadron Collider Physics-LHCP2020

25-30 May, 2020

online

\footnotetext{
${ }^{*}$ Speaker
} 


\section{Introduction}

The ALICE Collaboration has built a dedicated heavy-ion detector to exploit the unique physics potential of nucleus-nucleus interactions at LHC energies. The aim is to study the physics of strongly interacting matter at extreme energy densities, where the formation of a new phase of matter, the Quark-Gluon Plasma (QGP), is expected. During Run 2 of the LHC the ALICE Diffractive (AD) detector was installed to extend the pseudorapidity coverage of ALICE, increasing its capacities to trigger diffractive and ultra-peripheral events, and during the Long Shutdown 2 (LS2) will be replaced for the Forward Diffractive Detector (FDD) to fulfill the new requirements of the LHC conditions. After the LS2, the LHC will progressively increase its luminosity and collision rate reaching up to about $50 \mathrm{kHz}$ and $6 \times 10^{-27} \mathrm{~cm}^{2} s^{-1}$ for $\mathrm{Pb}-\mathrm{Pb}$; and $1 \mathrm{MHz}$ and $10^{-34} \mathrm{~cm}^{-2} \mathrm{~s}^{-1}$ for pp collisions. To exploit the new LHC scenarios, ALICE will have new detectors [1] like the Muon Forward Tracker and the Fast Interaction Trigger (FIT) as well as a new continuous trigger mode in addition to the traditional trigger scheme at its disposal. The FIT system [2] is formed by three subdetectors: FV0, FT0 and FDD, which are the upgrades of the V0, T0 and AD detectors, respectively. The three subdetectors will be placed in the forward regions with respect to the interaction point. All three detectors are integrated in a common Front End Electronics and Detector Control System. The subdetectors of FIT will deliver the lowest trigger level and different trigger classes: minimum latency interaction trigger, luminosity monitoring, precision collision time and vertex, and determination of both, centrality and the event plane for heavy-ion collisions. The pseudorapitity coverage of FDD will allow it to have an important contribution to the monitoring of the LHC background and to provide vetoes for ultra-peripheral [3], electromagnetic and diffractive interactions.

\section{The FDD concept}

The FDD detector consist of two sub-detectors, placed in the A and C sides of the ALICE cavern along the beam pipe at 17 and 19.5 meters from the interaction point respectively (see Fig. 1). Each sub-detector or station is an assembly of two layers segmented in four pads. The pads are made of plastic scintillator BC-420, two wavelength shifting (WLS) bars coupled in two sides of the scintillators to transport the light to a PMT via a bundle of 192 fibres (96 for each WLS bar). The A- and C-side pads differ only in their inner corner due to the difference in the beam-pipe radius on their corresponding sides.

Comparing the signal time width of FDD with respect to $\mathrm{AD}$, a reduction from $\sim 40$ ns to $\sim 29$ ns is achieved by using materials with a better timing response in the construction of the pads. The main contribution is coming from the the NOL-38 WLS bar of FDD, that have a reduced decay time of about $1 \mathrm{~ns}$ in comparison with the $8.5 \mathrm{~ns}$ decay time of the EJ-289 WLS bar of AD. The plastic scintillator was changed to the BC-420 model, in order to match the absorption wavelength of the WLS bar. The optical fibers will be the sames as for AD (Kuraray PSM-Clear) and the PMT will be changed to a fine mesh 19-dynodes produced by Hamamatsu (H8409-70). Additionally, FDD will have a dedicated laser calibration system. It will be used for amplitude and time calibration and alignment, quality assurance, and monitoring of the gain and ageing of the detector. 


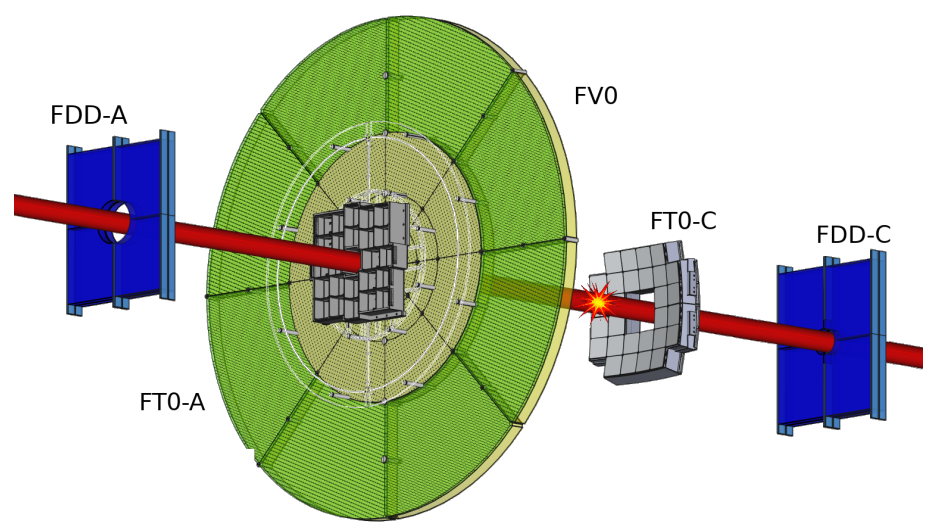

Figure 1: (Color online) Scheme of the FIT detector. The placement of the FDD with respect to the FV0 and FT0 subdetectors is shown (not to scale).

\section{Comments and outlook}

Based on the experience of working with the AD detector and its performance, it is expected that the FDD will be successfully constructed by the end of 2020 and the beginning of 2021 where its commissioning will start. With the actual scheme of forward detectors, FDD will make an essential contribution in beam monitoring tasks [4], in addition to the contributions to the studies of diffraction [5] and ultra-peripheral collisions [3]. The new materials used for the construction show a significant improvement in performance with respect to the AD. The full characterization and construction are in progress, including the tuning of the Front End Electronics and Detector Control System. The laser calibration system will allow for the monitoring of the detector to adjust parameters to guarantee the best performance.

Acknowledgements This research was partially funded by the Ministry of Education, Youth and Sports of the Czech Republic grant number LTT17018.

\section{References}

[1] ALICE Collaboration, Upgrade of the ALICE Experiment: Letter Of Intent. J. Phys. G41, 2014. doi.org/10.1088/0954-3899/41/8/087001.

[2] Trzaska, W. H. for the Alice Collaboration, New Fast Interaction Trigger for ALICE. Nuclear Instruments and Methods in Physics Research Section A, vol. 845, 2017. doi.org/10.1016/j.nima.2016.06.029.

[3] Contreras, J.G. and Tapia Takaki, J.D., Ultra-peripheral heavy-ion collisions at the LHC. Int. J. Mod. Phys. A 30, 1542012 (2015). doi.org/10.1142/S0217751X15420129.

[4] Gibson, Stephen et al., Beam-Gas Background Observations at LHC (2017). doi.org/10.18429/JACoW-IPAC2017-TUPVA032. 
[5] ALICE Collaboration, Measurement of inelastic, single- and double-diffraction cross sections in proton-proton collisions at the LHC with ALICE Eur. Phys. J., vol. 73, 2013. doi.org/10.1140/epjc/s10052-013-2456-0. 